\title{
An X-ray Optical Method for the Determination of Diffusion Properties in Very Thin Bimetallic Films
}

\author{
A. Wagendristel \\ Institute for Applied Physics, Technical University, Vienna, Austria
}

(Z. Naturforsch. 30 a, 1648-1654 [1975] ; received September 23, 1975)

\begin{abstract}
The change of $\mathrm{x}$-ray optical properties during diffusional alloy formation can be used for the evaluation of diffusion parameters in bimetallic thin film couples. The most pronounced phenomenon governed by these properties are the known Kiessig interferences which can be observed in the specular scattered $x$-ray beam at very small glancing angles. From these interference patterns diffusion data can be obtained by a comparison with calculated reflection curves based on a proper model for diffusion.

The most useful thickness range for this method is below $1000 \AA$, where common $x$-ray techniques lose their applicability. Since the changes in the interference patterns are most significant in the early diffusion stages, this technique is most suitable for observing initial interdiffusion, which very often occurs with enhanced diffusion rates.

The experimental check of the method is performed in the Au-Ag system. Bimetallic films of the pure components were annealed at temperatures between 200 and $300{ }^{\circ} \mathrm{C}$. In this temperature range diffusion parameters from $10^{-16}$ to $10^{-14} \mathrm{~cm}^{2} / \mathrm{s}$ were found in good agreement with values obtained by other techniques.
\end{abstract}

\section{Introduction}

In several comprehensive reviews the peculiarities of thin film diffusion have been reported ${ }^{1-3}$. One of these individualities is the dependence of the diffusion parameters upon annealing time caused by the reduction of the initially high defect concentration during annealing ${ }^{2,4}$. In order to observe this effect it is advantageous to follow the diffusion process continuously during the annealing treatment or at least to examine the same sample during a stepwise anneal. In such cases non-destructive methods must be employed, which in general require already some knowledge of the diffusion process. This knowledge is necessary for the establishment of proper models for diffusion, since diffusion parameters are mostly deduced from a comparison of calculated and measured values obtained as an integral property over the film volume or the film surface.

In the present paper an $x$-ray technique for the observation of interdiffusion in very thin films is presented ${ }^{*}$. Apart from common diffraction methods $^{5-7}$ and emission-absorption techniques ${ }^{8,9}$ the change of the scattering properties for x-rays under very small angles of incidence and reflection is related to the stage of diffusion. In analogy to common optical interferences originated in thin media $\mathrm{x}$-ray interferences can be observed in the beam

* In a paper recently presented at the $3^{\text {rd }}$ ITFC ${ }^{18}$ some results obtained by this method have been reported. A detailed description of the method, however, was announced to be published separately. reflected specularly on thin films close to the angle for total external reflection. From the angular positions of the intensity extrema the thickness of the film as well as its refractive properties for the monochromatic x-rays used can be evaluated ${ }^{10-12}$. In addition several authors have attempted to deduce surface or interface properties of thin films from the shape of the reflection curve ${ }^{13-17}$.

Considering multilayer systems whose components are able to interdiffuse we have to expect a significant change in the interference pattern during this process, since the different atoms change their position within the film and therefore the amplitude as well as the phase of their scattered radiation. This effect can be clearly envisaged by considering a bimetallic film in the initial as well as in the totally homogenized stage. According to the three interfaces originally present we obtain an interference pattern corresponding to three reflected beams whereas the final stage shows two beam interferences generated only by the film surface and the substrate interface reflection. The method based on these considerations can be applied in a thickness range from several 100 up to $1000 \AA$, where Kiessig interferences are most pronounced. Similarly to other indirect methods the diffusion stage in the sample is determined by a comparison of calculated and measured reflection curves. It is therefore necessary first to describe the theoretical background of the calculation of reflection curves as well as the special features of diffusion in polycrystalline films. 


\section{Theoretical Considerations}

\section{Calculation of reflection curves}

The angular dependence of the intensity of monochromatic x-rays scattered by thin films can be calculated by several formalisms. A straight forward treatment is possible by adopting the small angle scattering theory, in which the layer is considered as an assembly of coherent scattering elements. The scattering properties of each element are given by its particular composition. Since the boundaries of the scattering medium are smooth and the angle of incidence is very small we also have to account for the refraction of incident and scattered radiation.

A second possibility for calculation is the application of Fresnel's formulae to the film interfaces. In this case we have to approximate a diffusion zone by a stack of layers with uniform concentration. When the beam passes a boundary plane the Fresnel formulae determine the transmitted and the reflected part. Neglecting all reflections of higher order we then obtain a bundle of waves reflected at each boundary and weakened by secondary reflection and absorption before having reached the surface. The sum of all scattered waves finally gives the reflected beam.

A third method proposed by Parratt ${ }^{13}$ is a strict application of Maxwell's equations to boundary planes in layered systems.

In a further paper ${ }^{19}$ it will be shown in detail that all theories mentioned above give identical results in an angular range apart from the angle for total reflection. But, as we shall see later it is advantageous to use the low order reflections for the observation of the diffusion process. These reflections occur next to or even in the range of total reflection for one component. Since the small angle scattering theory is therefore not suitable Parratt's formalism was used, which is briefly reviewed.

We consider a beam of wavelength $\lambda_{0}$ incident to a boundary plane $z_{n}=$ const separating the $n^{\text {th }}$ and the $n-1^{\text {st }}$ layer of a laminated system. The electric vector in the $n^{\text {th }}$ layer is given by

$\boldsymbol{E}_{n}(x, z, t)=\boldsymbol{E}_{n 0} \cdot \exp \left\{i\left[\omega t-\left(k_{n x} x+k_{n z} z\right)\right]\right\}$

where $k_{n x}$ and $k_{n z}$ are the components of the wave vector and $\boldsymbol{E}_{n 0}$ is the amplitude of the beam after having passed the boundary at $z_{n} . x$ and $z$ are spacial coordinates. For low angles of incidence the wave vector can be approximated by

$$
\left.\begin{array}{c}
k_{n x} \doteq k_{(n-1) x} \doteq k_{1} \\
k_{n z} \doteq k_{1} \cdot\left[\Theta^{2}-2(\delta+i \beta)_{n}\right]^{1 / 2} \doteq k_{1} \cdot f_{n} .
\end{array}\right\}
$$

$\Theta$ is the glancing angle and $(\delta-i \beta)_{n}$ are the decrements of the refractive index in the layer $n$ given by

$$
\delta \doteq 1.36 \cdot \lambda_{0}{ }^{2} \cdot \varrho \cdot 10^{-6}
$$

and

$$
\beta \doteq \mu \lambda / 4 \pi .
$$

$\varrho$ is the mass density and $\mu$ is the linear absorption coefficient. Using

$$
a_{n}=\exp \left(-i k_{1} f_{n} d_{n} / 2\right)=\exp \left(-i f_{n} d_{n} / \lambda_{0}\right),
$$

where $d_{n}$ is the thickness of the layer $n$, we express the condition of continuity for the tangential component of the electric vector at the boundary between the $n-1^{\text {st }}$ and the $n^{\text {th }}$ lamina:

$$
\begin{array}{r}
a_{n-1} E_{n-1}+a_{n-1}^{-1} E_{n-1, R}=a_{n}^{-1} E_{n}+a_{n} E_{n, R}, \\
\left(a_{n-1} E_{n-1}-a_{n-1}^{-1} E_{n-1, R}\right) f_{n-1} k_{1} \\
=\left(a_{n}^{-1} E_{n}-a_{n} E_{n, R}\right) f_{n} k_{1} .
\end{array}
$$

At this plane 4 beams are present, each represented by a corresponding term in (5) and (6). The subscript $R$ is assigned to those waves advancing in the direction of reflection. $E_{n}$ are the values of the electrical field strength in the middle of the layer $n$. The ratio of the difference and the sum of (5) and (6) leads to a recursion formula for $E_{n}$ and $E_{n, R}$

$$
\begin{aligned}
& R_{n-1, n}=a_{n-1}\left[\frac{R_{n, n+1}-F_{n-1, n}}{R_{n, n+1} F_{n-1, n}+1}\right] \\
& R_{n, n+1}=a_{n}{ }^{2}\left(E_{n, R} / E_{n}\right), \\
& F_{n-1, n}=\left(f_{n-1}-f_{n}\right) /\left(f_{n-1}+f_{n}\right) .
\end{aligned}
$$

The reflection coefficient $R_{1}=E_{1, R} / E_{1}$ representing the reflectivity of the whole stack is evaluated in a step wise manner beginning from the film substrate interface. This plane is characterised by $R_{n, n+1}=0$ since the beam undergoes no further reflection after having passed through.

\section{Diffusion model}

The application of the theory described above requires the diffusion zone to be approximated by a stack of layers showing uniform concentration. If we consider systems of miscible components and approximate the diffusion profile by solutions of Fick's second law we obtain for the concentration 
$c($ vol-\%) in a given depth $z$

$c(z, t)\left\{\begin{array}{c}=A_{n 0} \exp \left(-D t n^{2} \pi^{2} / d^{2}\right) \\ \cdot \cos (n \pi z / d) \ldots 0 \leqq z \leqq d, \\ \equiv 0 \ldots \text { elsewhere, }\end{array}\right.$

where $A_{n 0}$ are the Fourier coefficients of the original step profile, whereas $d$ is the total thickness, $D$ the diffusion coefficient and $t$ the diffusion time. The validity of this one dimensional approach, however, is somewhat questionable, since thin films are generally intersected by a fine-meshed network of grain boundaries, giving rise to short circuit diffusion along them. A rather inhomogeneous diffusion zone has to be expected in this case. If structural data of the films are available it is possible to simulate the spatial diffusion profile as shown in Figure 1. From this picture, giving an impression of

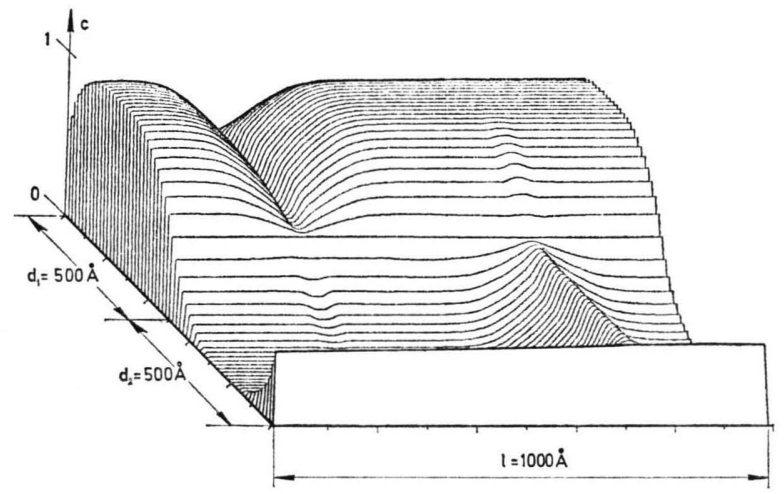

Fig. 1. Spatial distribution of the concentration in a thin film diffusion couple obtained by computer simulation ${ }^{20}$. Data for computation: $d_{1}=d_{2}=500 \AA$, cut out part of $l=1000 \AA$ length, two displaced grain boundaries perpendicular to the surface. Diffusion coefficients $D_{\mathrm{b}}=10^{-14}$, $D_{\mathrm{B}}=1.7 \cdot 10^{-10}, D_{\mathrm{gb}}=5 \cdot 10^{-10} \mathrm{~cm}^{2} \mathrm{~s}^{-1}$ for the bulk, surface and the grain boundaries resp., $t=25 \mathrm{~s}$.

short circuit diffusion along the interface, the surfaces and the grain boundaries, the following conclusion may be drawn: Since uniform concentration is almost instantaeously present in the short circuits the flux of atoms into the lattice is determined mainly by the area of short circuits. In polycrystalline films the grain boundary area is generally much larger than the area of the interface. Hence we have to expect that the number of atoms moving into the lattice of the partner via grain boundaries exceeds by far that being incorporated directly through the interface. In order to account for this predominant mechanism, we propose an approximate diffusion model, which has already been used for the resistometric investigation of diffusion ${ }^{4}$. In this model the direct exchange of atoms through the interface is neglected. The homogenisation is assumed to occur only by lateral diffusion from the grain boundaries into the bulk. This model leads to a step profile since the lateral diffusion flux is independent of the coordinate $z$ normal to the film plane. The step height decreases as diffusion proceeds. In this case we may define an over-all diffusion parameter as that value of the one dimensional approach, which would lead to the same mean concentration in each individual layer.

The diffusion profile is then given by

$$
\bar{c}(z, t)\left\{\begin{array}{l}
=1 / d_{1} \int_{0}^{d_{1}} c(z, t) \mathrm{d} z, \ldots 0 \leqq z \leqq d_{1}, \\
=1 / d_{2} \int_{d_{1}}^{d} c(z, t) \mathrm{d} z, \ldots d_{1} \leqq z \leqq d, \\
\equiv 0, \quad \ldots \text { elsewhere }
\end{array}\right.
$$

where $c(z)$ is connected to the diffusion parameter $D$ by (10). A comparison of both models is shown in Figure 2. Both expressions for the diffusion profile are valid for $t=0$ and $t \doteq z^{2} / D$ at which time the film is nearly totally homogenized. The actual intermediate stages of diffusion will show a concen-
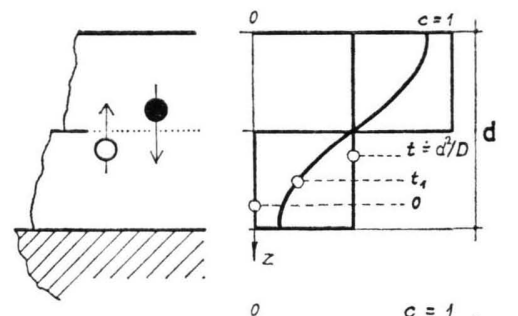

a)
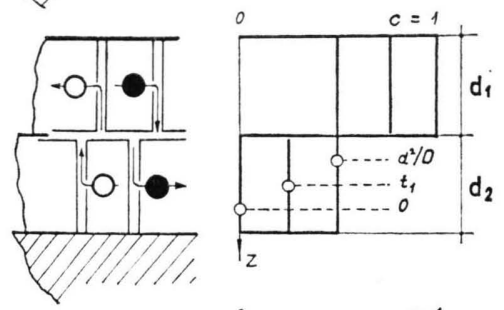

b)
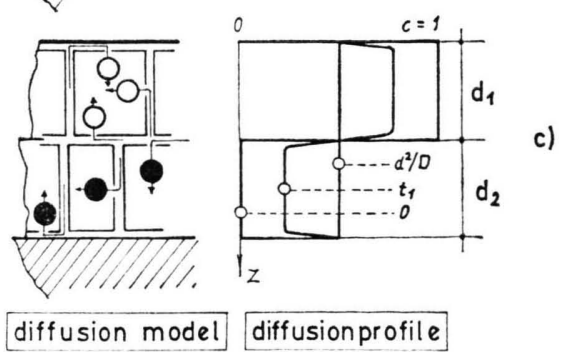

Fig. 2. Comparison of two approximate models for diffusion with real diffusion in a thin film couple. a) One dimensional approach, b) Grain boundary approximation, c) Real process. 
tration profile between the two limiting models represented by (10) and (11). In the following investigation we have used both models as basis for the calculation of reflection curves for different stages of interdiffusion. A decision to which approximation the true concentration profile tends can be taken from the shape of the reflection curves to be shown later.

\section{Experiment}

For convenience the system Ag-Au was used to check the method. Both components can by easily evaporated and differ significantly in density and atomic weight thus showing different $\mathrm{x}$-ray optical properties. Furthermore the effect of decreasing diffusivity with anealing time is already established for this system ${ }^{4,21}$.

\section{Preparation of samples}

Bimetallic films of the pure components were produced by subsequent evaporation from tungsten boats during the same pump-down. Polished glass, cleaned by means of an ultrasonic treatment in several fat solvents was used as substrate. The pressure during the evaporation was in the range of $10^{-5}$ torr. The thickness as well as the deposition rate were controlled by a quartz crystal balance. Accurate thickness measurements, however, were carried out directly on the samples by fitting computed $\mathrm{x}$-ray reflection curves to the measured values. The deposition sequence was chosen so that most significant changes in the interference pattern during diffusion could be expected. A simple consideration shows that this condition is best satisfied when the material with the lower decrement of the refractive index is on top of the layer with the higher one. In this case single film reflections of the top layer can be observed in an angular region where the bottom layer is still opaque because of total reflection. The theory predicts the disappearence of these reflections with proceeding diffusion. In a number of experiments $200 \AA$ of $\mathrm{Ag}$ on top of a $600 \AA$ layer of gold was found to be an optimum combination, since only one strong interference of the silver layer is observed in the range of total reflection for gold.

In order to prevent the samples from being damaged during the annealing procedures the film couples were covered with a $100 \AA$ film of $\mathrm{MgF}_{2}$. This layer does not influence the diffraction pattern, since the decrement of its refractive index and the thickness are very small.

\section{Annealing treatment}

In order to avoid uncontrolled heating with long heating up periods the samples were not annealed in vacuum. The covering $\mathrm{MgF}_{2}$ layer allowed the heat treatment to be performed under atmosphere, where an almost instantaneous temperature rise was achieved by pressing the sample with the film side against a heated copper block. The same procedure was carried out with a copper block of room temperature in order to quench the film after the annealing. Temperatures of 200 to $300{ }^{\circ} \mathrm{C}$ were chosen. The intervals of the annealing ranged from $15 \mathrm{~s}$ to several hours.

\section{3. $x$-ray Investigations}

The x-ray experiments were performed in a Philips diffractometer. $\mathrm{Cu}-\mathrm{K}_{\alpha}$ radiation selected with a counter tube monochromator was used. In order to obtain a reference intensity for the comparison of different reflection curves we started the measurements from an angle below the limit for total reflection of the $\mathrm{Ag}$ layer. Integrated pulse rates we recorded as a function of rising glancing angle. The beam divergence was estimated from the distance focus - counter slit and its width to be $0,02^{\circ} 2 \Theta$. It was accounted for by convolution of the reflection curves with a square function of the same breadth ${ }^{22}$.

Measurements of the thickness as well as the refractive properties were carried out by means of Kiessig's method ${ }^{12}$ on the single layers deposited separately next to the couples. As known from several other works ${ }^{23}$ the refractive properties differed markedly from the values for the bulk material obviously as a result of reduced mass density. With these values the reflection curves of the diffusion system in the initial stage were calculated. Small corrections in thickness and decrement were necessary to obtain best agreement with the measured curves.

\section{Results}

\section{Computation}

The following data are processed: $\delta(c), \beta(c), c$, $d_{\mathrm{Ag}}, d_{\mathrm{Au}}, \delta_{\text {substrate }}$ and $\beta_{\text {substrate }}$. The concentration dependence of $\delta$ and $\beta$ in the system Ag-Au is fairly linear between the values of the components. In the one dimensional approach the diffusion profile represented by (10) was approximated by 100 steps of uniform concentration. Plots of the calculated 


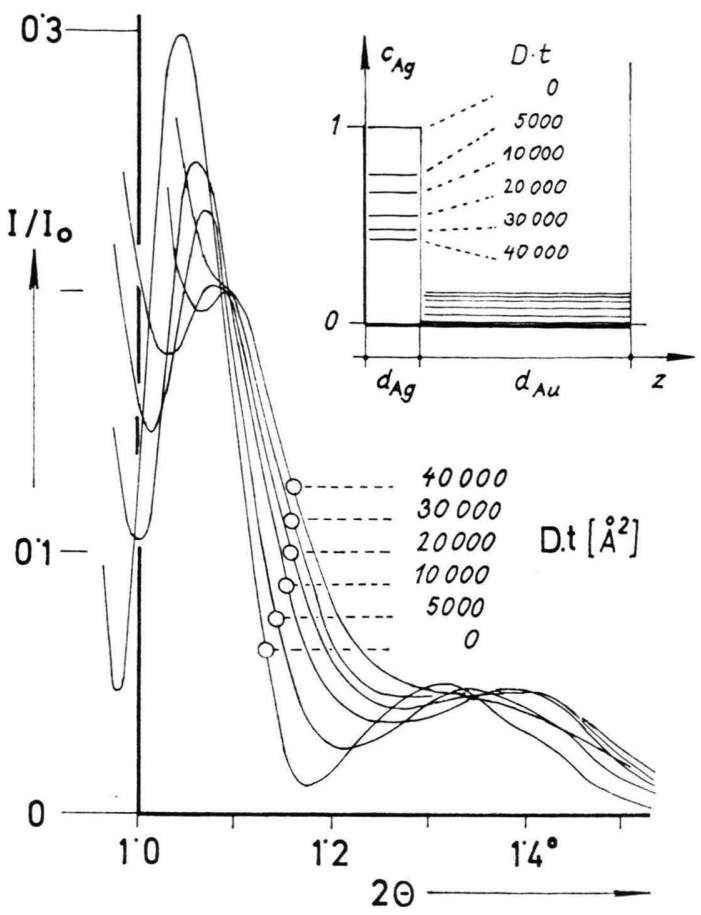

a)

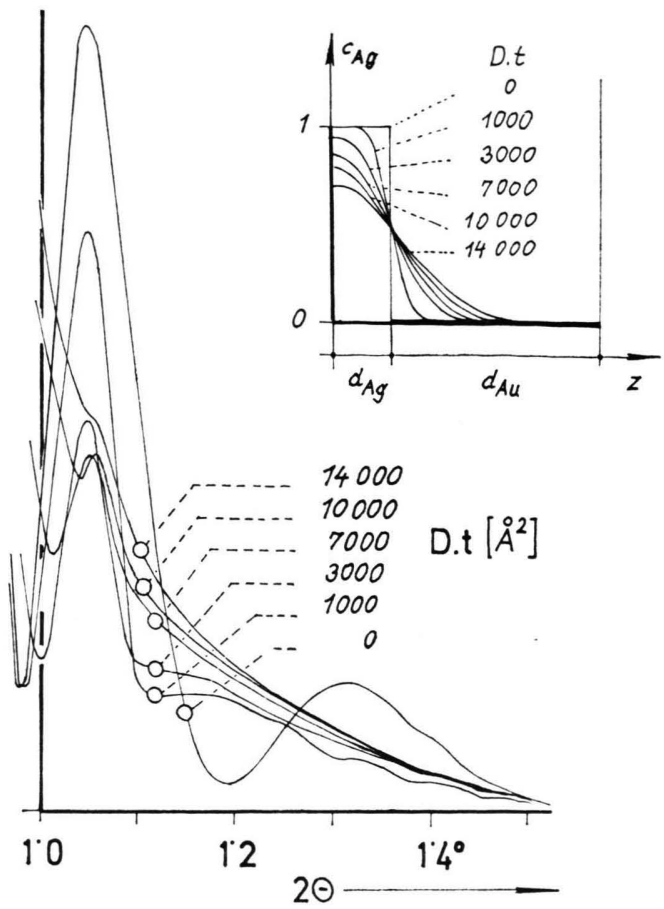

b)

Fig. 3. Reflection curves calculated for several diffusion stages in a couple consisting of $173 \AA$ Ag on top of $650 \AA$ Au. a) Grain boundary approach, b) One dimensional approximation. ( $\varrho_{\mathrm{Au}}=45 \cdot 10^{-6}, \beta_{\mathrm{Au}}=3,5 \cdot 10^{-6}, \varrho_{\mathrm{Ag}}=30^{\circ} \cdot 10^{-6}$, $\left.\beta_{\mathrm{Ag}}=2,9 \cdot 10^{-6},\right)$

reflection curves are shown in Fig. $3 \mathrm{~b}$ for the one dimensional approach and in Fig. 3 a for the grain boundary model. The parameter $D \cdot t$ represents different stages of diffusion. A comparison of both series shows marked differences in shape as well as in the value $D \cdot t$ assigned to similar curves.

\section{Measurement}

A series of reflection curves typical for several diffusion stages at different annealing temperatures is presented in Figure 4. From the excellent agreement of the shape of the curves with that calculated on the basis of the grain boundary diffusion model it can be concluded that the actual process of interdiffusion is in fact dominated by the lateral lattice diffusion out of grain boundaries. This is supported by electron micrographs of the films which exhibited a grain size below $150 \AA$, thus giving a grain boundary area approximately twenty times larger than the interface area and in addition a ratio of $1 / 5$ of the actual lateral diffusion path to the film thickness. Attempts to refine the grain boundary diffusion model by rounding off the sharp concentration steps at the interfaces did not change the reflection curves very much, but a slightly larger decrease of the interference maxima was observed. This decrease, however, was not sufficient to give a good match of measured and calculated reflection -curves in the intensity scale. The rapid decay of the interferences during the very first annealing period ( $15 \mathrm{~s}$ ) could not be achieved by changing the input data of the computation. So we have to assume that it is the result of the inhomogeneity in the diffusion zone with behaviour similar to that of a rough surface. This interpretation, however, has still to be checked by recording rocking curves of the samples which should show an enhanced part of the non-specular scattered x-rays ${ }^{22}$.

Effective diffusion parameters $D$ were deduced by comparing measured and calculated reflection curves. These data are shown in Figure 5. Since the diffusion parameter is not constant with time we have to replace $D \cdot t$ in (10) by $\int_{0}^{t} D\left(t^{\prime}\right) \mathrm{d} t^{\prime}$ which means that the values $D$ are understood as a time average be- 


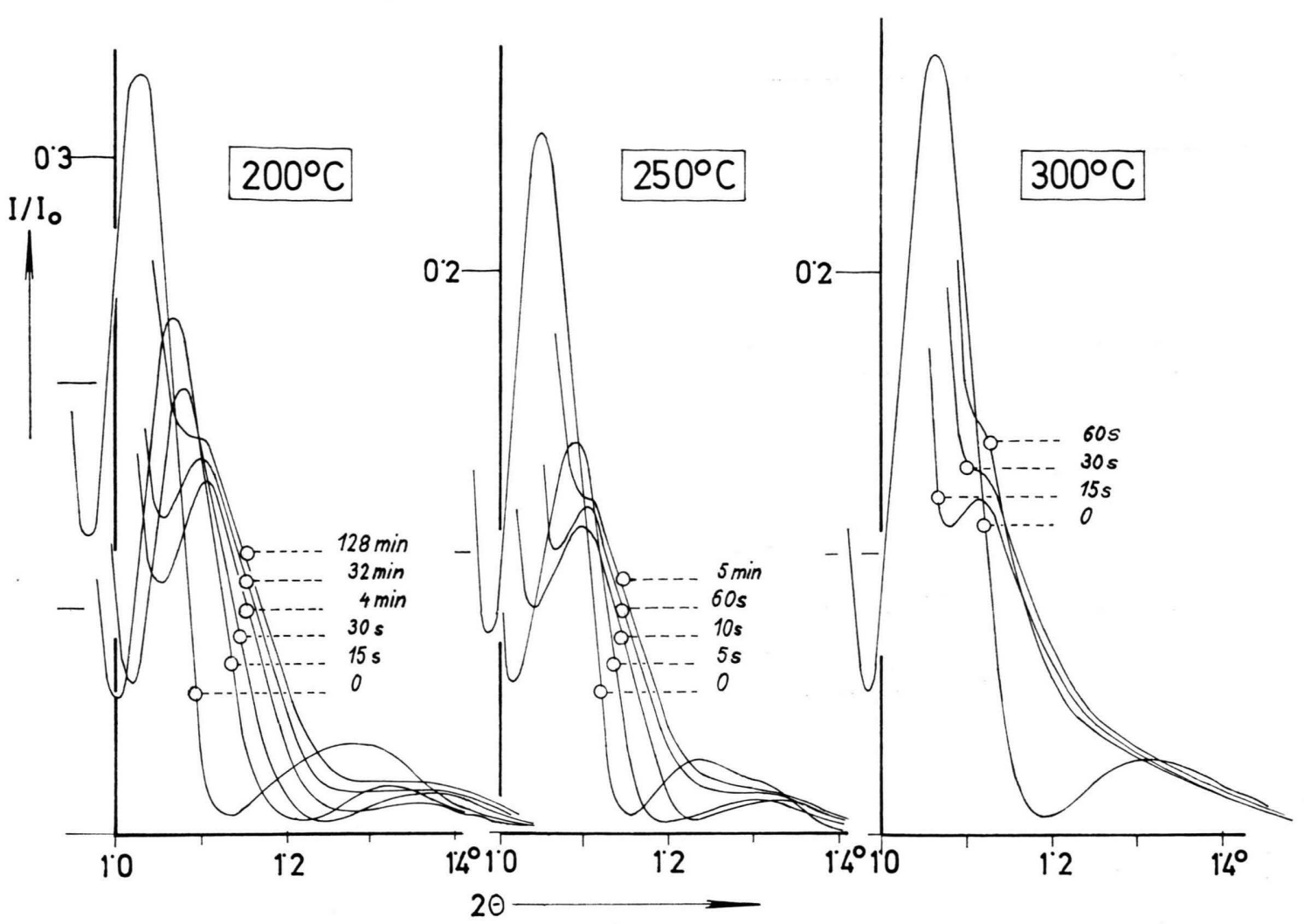

Fig. 4. Measured reflection curves consisting of $173 \AA \mathrm{Ag}$ on top of $650 \AA \mathrm{Au}$ in various annealing stages.

tween $t^{\prime}=0$ and $t$. In the diagram diffusion parameters obtained by resistivity measurements ${ }^{4}$ are also presented. The values $D$ as well as their time dependence are in very good agreement with the data found by the x-ray method.

A rapid initial decrease of $D$ is characteristic for all annealing temperatures. It can be explained by the reduction of the excess density of vacancies quenched in during condensation. Because of their small life time they escape quickly to grain boundaries or free surfaces during the first annealing period. This migration is accompanied by an enhanced flux of hetero-diffusion as well. The further decay of $D$ is less pronounced and possibly due to the reduction of the diffusional short circuits. An interesting explanation of the decreasing diffusivity was given by Cook and Hilliard ${ }^{21}$, who made the same observation in the $\mathrm{Ag}-\mathrm{Au}$ system. These authors observed enhanced diffusion only during the recrystallisation period of the film and concluded that advancing grain boundary short circuits are much more effective than stable ones. Recrystallisa- tion, however, is a process less fast than the annihilation of vacancies, so that a contribution of excess vacancies to the enhanced diffusion flux was excluded in that paper. In order to check this interpretation a gold film was annealed for $30 \mathrm{~s}$ at $150^{\circ} \mathrm{C}$ before it was covered with $\mathrm{Ag}$ and $\mathrm{MgF}_{2}$. The diffusion parameters obtained from the reflection curves are also shown in Fig. 5 together with the data of the freshly condensed couples. A reduction of more than one magnitude in connection with a rather weak time dependence of $D$ is the result of the preannealing of the gold film. We therefore tend to the opinion of Weaver ${ }^{2}$ who attributes the very fast initial diffusion to excess vacancies. The decay of the diffusion coefficient at longer annealing times, however, is possibly caused by a reduction as well as a stabilisation of the diffusional short circuits.

The usual way to identify the diffusion mechanism dominating a homogenisation process is the measurement of the activation energy of the diffusion coefficient. Values in the range approximately $40,000 \mathrm{kcal} / \mathrm{mol}$ are attributed to pronounced volume 


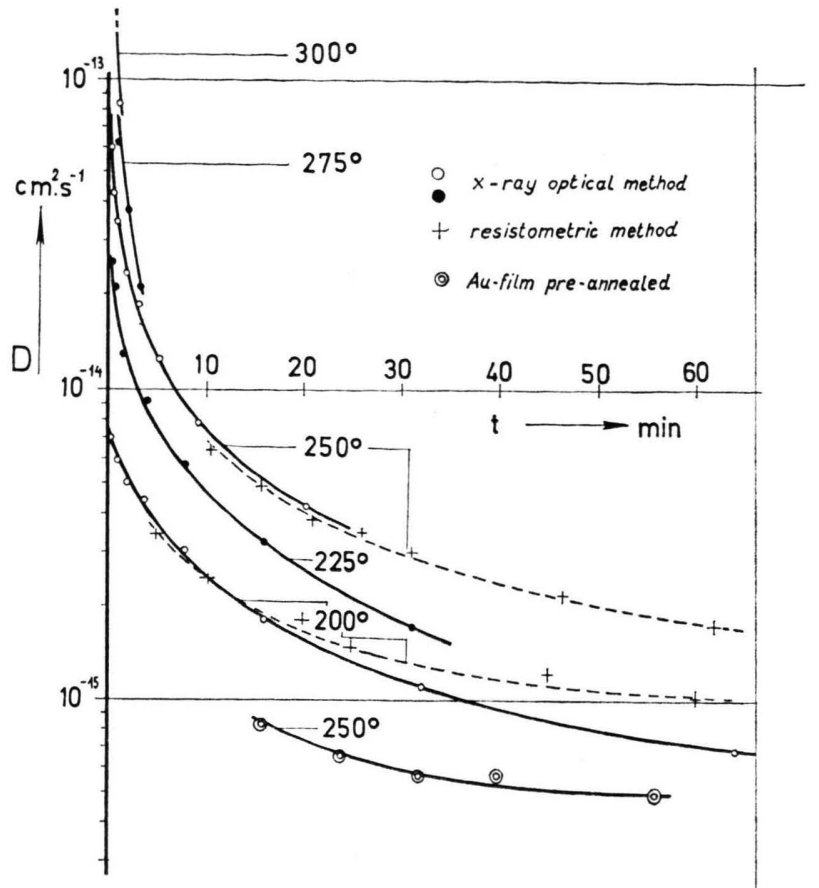

Fig. 5. Mean diffusion parameters $D$ as a function of annealing time.

diffusion whereas values around $20,000 \mathrm{kcal} / \mathrm{mol}$ indicate a strong regime of grain boundary diffusion. The application of such a procedure for very thin films is questionable, since grain boundaries are filled with the partner atoms almost immidiately.

1 C. Weaver and R. M. Hill, Adv. Phys. 8, 375 [1959].

2 C. Weaver, Physics of Thin Films, 6, 301, Acad. Press, London 1971.

3 A. Gangulee, P. S. Ho, and K. N. Tu, Low Temperature Diffusion and Application to Thin Films, Elsevier Sequoia, Lausanne 1975.

4 A. Wagendristel, Phys. Stat. Sol. (a) 13, 131 [1975].

5 C. R. Houska, Thin Solid Films 25, 451 [1975].

6 K. N. Tu and B. S. Berry, J. Appl. Phys. 43, 3283 [1972].

7 A. Wagendristel, Appl. Phys. 7, 175 [1975].

${ }^{8}$ H. Ebel and A. Taurok, Spectr. Chim. Acta 27 b, 415 [1972].

9 R. Feldkamp and G. Schimmel, Z. Metallkde. 54, 186 [1963].

10 H. Kiessig, Ann. Phys. 10, 729 [1931].

11 R. Riedmüller, Ann. Phys. 20, 377 [1934].

12 W. Umrath, Z. Angew. Phys. 22, 46 [1967].
Furthermore the conditions under which the diffusion process takes place change with diffusion time as indicated by the reduction of $D$. So, the question arises which values shall be compared. In the present case fortunately the method of investigation is very sensitive to the type of homogenisation as established by the above measurements.

\section{Conclusion}

The method described above has been shown to be a useful tool for the investigation of diffusion in very thin films $(\leqq 1000 \AA)$. As this method is very sensitive to small changes in the spatial distribution of different scattering atoms within the film a separation of a proper model from others less suitable is possible. The information about the diffusion process is therefore not confined to the description of the stage of homogenisation by an over all diffusion parameter. From the model employed we may also determine the type of interdiffusion.

\section{Acknowledgements}

The author is indebted to Mr. D. Wolf for computation of the reflection curves and Dr. H. Bangert for discussion. This work was supported by the Austrian Fond zur Förderung der Wissenschaftlichen Forschung.

13 L. G. Parratt, Phys. Rev. 95, 359 [1954].

14 N. Wainfan, N. J. Scott, and L. G. Parratt, J. Appl. Phys. 30, 1604 [1959].

15 N. Wainfan and L. G. Parratt, J. Appl. Phys. 31, 1331 [1960].

16 W. Petzold, Z. Angew. Phys. 15, 525 [1963].

17 G. Kühnen, Optik 26, 582 [1967/1968].

18 A. Wagendristel, D. Wolf, and H. Bangert, Proc. $3^{\text {rd }}$ Intern. Conference on Thin Films, Elsevier, Lausanne.

19 A. Wagendristel and D. Wolf, to be published.

20 A. Wagendristel, E. Tschegg, E. Semerad, and H. Bangert, Surface Science, submitted for publication.

21 H. E. Cook and J. E. Hilliard, J. Appl. Phys. 40, 2191 [1969].

22 J. P. Sauro, J. Bindell, and N. Wainfan, Phys. Rev. 143, 439 [1966].

23 A. Wagendristel and E. Söllner, Z. Naturforsch. 30 a, 44 [1975]. 